\title{
Statistical properties of genetic learning in a model of exchange rate
}

\author{
Jasmina Arifovic ${ }^{\mathrm{a}, *}$, Ramazan Gençay ${ }^{\mathrm{b}, \mathrm{c}, \mathrm{d}}$ \\ ${ }^{a}$ Department of Economics, Simon Fraser University, Burnaby, BC, Canada V5A 1 N6 \\ ${ }^{\mathrm{b}}$ Department of Economics, University of Windsor, Windsor, Ont. Canada N9B 3P4 \\ ${ }^{\mathrm{c}}$ Department of Economics, Bilkent University, Bilkent 06533, Ankara, Turkey \\ ${ }^{\mathrm{d}}$ Olsen \& Associates, Seefeldstrasse 233, Zurich, CH-8008, Switzerland
}

Received 1 August 1998; accepted 30 April 1999

\begin{abstract}
We study statistical properties of the time series of the exchange rate data generated in the environment where agents update their savings and portfolio decisions using the genetic algorithm. The genetic algorithm adaptation takes place within an overlappinggenerations model with two currencies and the free-trade, flexible exchange rate system. The theoretical model implies a constant exchange rate under the perfect foresight assumption. Under the genetic algorithm learning, the model's equilibrium dynamics is not constant but exhibits bounded oscillations. The time series analysis of the data indicates that the dynamics of the exchange rate returns is chaotic. Out-of-equilibrium inequality of rates of return on two currencies prompts the genetic algorithm agents to take advantage of the arbitrage opportunities by increasing the amount of the currency with higher rate of return in their portfolios. This profit seeking results in chaotic patterns of the exchange rate series. (C) 2000 Elsevier Science B.V. All rights reserved.
\end{abstract}

\section{Introduction}

The genetic algorithm has been increasingly employed to model the behavior of economic agents in macroeconomic models. A partial list of references

\footnotetext{
* Corresponding author.
} 
includes Arifovic (1995,1996,1998), Arifovic et al. (1997), Bullard and Duffy (1998a,b), Marimon et al. (1990), Dawid (1996). The genetic algorithm learning has been used both as an equilibrium selection device and as a model of transitional, out-of-equilibrium dynamics.

When used in economic modeling, the genetic algorithm describes the evolution of a population of rules, representing different possible beliefs, in response to experience. In a parallel to population genetics, these rules undergo a selection process whereby more successful ones become more numerous in the population. The rules are subjected to random mutations and to recombination of their parts. In turn, such newly created rules contribute to the diversity of the population.

There are several advantages in modeling of agents' adaptation in this way. Genetic algorithms impose low requirement on the computational ability of economic agents. They allow for modeling the heterogeneity of agents' beliefs. Survival of decision rules depends on their performance, measured by the payoff that agents receive by employing them. Genetic algorithm patterns successfully mimic the behavior of human subjects in controlled laboratory settings (Arifovic, 1995,1996). ${ }^{1}$

We study the statistical properties of genetic algorithm learning in the overlapping-generations environment with two currencies and a free-trade, flexible exchange rate system. The supplies of both currencies are constant. The equilibrium exchange rate of this economy is constant and indeterminate. In this model (Arifovic, 1996) agents use the genetic algorithm to update their decisions regarding savings and the portfolio composition, i.e. what fraction of savings to place in each currency. Genetic algorithm simulations resulted in the persistent fluctuations of the exchange rate. The stability analysis showed that the stationary rational expectations equilibria of the two-currency model were unstable under the genetic algorithm dynamics. The out-of-equilibrium inequality of the rates of return on the two currencies drives the changes in the fractions of holdings of the two currencies that in turn drives the exchange rate fluctuations. At the same time, these exchange rate fluctuations were not accompanied by large oscillations of the first and the second-period consumption values, which remain close to the Pareto optimal, stationary equilibrium values. In this paper, we analyze the properties of the exchange rate time series.

The time series properties of the data generated in the genetic algorithm economies have not been examined using formal statistical tests. Our work represents the first step in devising a methodology for examining the properties

\footnotetext{
${ }^{1}$ Arthur et al. (1997) use an artificial intelligence algorithm which incorporates genetic algorithm for generation of new rules to simulate artificial stock market. Asset prices and trading volume show statistical features very similar to the statistical features of actual data. Arthur et al. (1998) has a detailed discussion of the time series properties of an artificial stock market.
} 
of the data generated in the genetic algorithm simulations. This should eventually lead to their comparison to the properties of the actual macroeconomic time series.

The properties of the exchange rate series indicate the existence of a chaotic attractor. A large number of simulations that we conducted for different sets of the overlapping-generations model parameter values and the genetic algorithm values show that this result is robust to changes in the parameter values. We provide the analysis of why the observed data is chaotic. In the framework that we study, it is the indeterminacy of the equilibrium exchange rate and exploitation of arbitrage opportunities that generate chaos. Out-of-equilibrium inequality of rates of return on two currencies prompts the genetic algorithm agents to take advantage of the arbitrage opportunities by increasing the amount of the currency with higher rate of return in their portfolios. This profit seeking results in chaotic patterns of the exchange rate series. Our analysis confirms that these dynamics do not depend on the specific parameter values.

While the model considered in this paper is not the perfect foresight one, the behavioral assumptions are explicitly laid out. The economic model is a model of fiat money based on microeconomic foundations. In addition, as other research has shown, the genetic algorithm adaptation generates behavioral patterns very similar to those observed in the experiments with human subjects. This sort of behavior performs much better in capturing the main features of the data generated in the laboratory experiments with human subjects than models based on the perfect foresight hypothesis or models that use other learning algorithms.

Other research has demonstrated that the genetic algorithm has very good global convergence properties. Therefore, the instability of stationary rational expectations equilibria under the genetic algorithm adaptation of this model is not the result of the genetic algorithm's general inability to converge. Its source is in the model's indeterminacy of equilibria that, in interaction with the genetic algorithm operators, gives rise to the persistent fluctuations of the exchange rate. $^{2}$

Thus the driving force of endogenous fluctuations in this model is the indeterminacy of equilibria. It is interesting to compare this to the other two classes of models with endogenous fluctuations that have been recently studied. Brock and Hommes (1997) study a cobweb type of model with heterogenous strategies in which it is more costly to obtain the rational expectations strategy than arbitrary prediction rules. They show that the evolutionary competition among the strategies may result in endogenous price fluctuations, and chaotic behavior for certain parameter values in an unstable cobweb economy.

\footnotetext{
${ }^{2}$ It is worthwhile to point out again that the savings decisions of the genetic algorithm agents remain very close to the stationary values after initial adjustment.
} 
Arthur et al. (1997) study an asset pricing model that has a constant fundamental rational expectations equilibrium and a multiplicity of rational expectations equilibrium paths where prices grow at the risk free rate of return. In their model, agents are endowed with heterogeneous predictor rules. The survival and selection of these rules depend on their previous periods' performance. Arthur et al. (1997) observe occasional complicated price fluctuations. However, they do not present numerical evidence of chaos.

Brock and Hommes (1998) analyzed the same asset pricing model with heterogenous beliefs and the evolutionary updating based on past performance. They show that complicated, chaotic price fluctuations can arise. These fluctuations are characterized by irregular switching between the constant fundamental rational expectations solution and the exploding bubble solutions.

The common feature of the above models that can generate endogenous fluctuations and our exchange rate model is that all of them are based on the evolutionary updating of agents' decision rules. The differences lie in the actual source of the fluctuations. Thus in the cobweb model with a unique rational expectations equilibrium, it is the costs of using rational expectations decision rules that are the source of complicated dynamics. In the asset pricing model, the multiplicity of rational equilibrium paths can give rise to complicated dynamics. In case of our exchange rate model, it is the indeterminacy of the rational expectations equilibria that is the source of the chaotic dynamics.

In Section 2, a two-currency, overlapping-generations economy and the genetic algorithm application are described. The time series properties of the genetic algorithm model are presented in Section 3. This section also contains the analysis and the explanation of why the observed dynamics occurs. We conclude thereafter.

\section{The economy}

The economy is a version of Karaken and Wallace (1981) two-country overlapping-generation model with a flexible exchange rate system and no restrictions on foreign currency holdings. At each date $t, t \geq 1$, there are born $N$ young people, in each country, said to be of generation $t$. They are young at period $t$ and old at period $t+1$. Each agent of generation $t$ is endowed with $w^{1}$ units of a single consumption good at time $t$ and $w^{2}$ of the good at time $t+1$, and consumes $c_{t}(t)$ of the consumption good when young and $c_{t}(t+1)$ when old. Agents in both countries have common preferences given by: $u_{t}\left[c_{t}(t), c_{t}(t+1)\right]=c_{t}(t) c_{t}(t+1)$.

An agent of generation $t$ solves the following maximization problem at time $t$ :

$$
\max c_{t}(t) c_{t}(t+1)
$$


subject to

$$
\begin{aligned}
& c_{t}(t) \leq w^{1}-\frac{m_{1}(t)}{p_{1}(t)}-\frac{m_{2}(t)}{p_{2}(t)}, \\
& c_{t}(t+1) \leq w^{2}+\frac{m_{1}(t)}{p_{1}(t+1)}+\frac{m_{2}(t)}{p_{2}(t+1)},
\end{aligned}
$$

where $m_{1}(t)$ is the agent's nominal holdings of currency 1 acquired at time $t, m_{2}(t)$ is the agent's nominal holdings of currency 2 acquired at time $t, p_{1}(t)$ is the nominal price of the good in terms of currency 1 at time $t$, and $p_{2}(t)$ is the nominal price of the good in terms of currency 2 at time $t$. Agent's savings, $s(t)$, in the first period of life, are equal to the sum of real holdings of currency 1 , $m_{1}(t) / p_{1}(t)$, and real holdings of currency $2, m_{2}(t) / p_{2}(t)$.

The exchange rate $e(t)$ between the two currencies is defined as $e(t)=p_{1}(t) / p_{2}(t)$. When there is no uncertainty in the model, an equilibrium condition requires equal rates of return on all assets. Thus, the requirement for the monetary equilibrium in which both currencies are valued is that the rate of return on currency 1 , and the rate of return on currency 2 have to be equal

$$
R(t+1)=\frac{p_{1}(t)}{p_{1}(t+1)}=\frac{p_{2}(t)}{p_{2}(t+1)}, \quad t \geq 1,
$$

where $R(t+1)$ is the gross real rate of return between $t$ and $t+1$. Rearranging Eq. (2), we obtain

$$
\frac{p_{1}(t+1)}{p_{2}(t+1)}=\frac{p_{1}(t)}{p_{2}(t)}, \quad t \geq 1 .
$$

It follows from Eq. (3) that the exchange rate is constant over time:

$$
e(t+1)=e(t)=e .
$$

Individual's savings $s(t)$ are derived from the agent's maximization problem and aggregate savings, $S(t)$, are equal to $2 N s(t)$. The equilibrium condition in the loan market requires that the aggregate real world money demand, given by the aggregate savings, equals real world money supply, i.e. that

$$
S(t)=N\left[w^{1}-w^{2} \frac{p_{1}(t+1)}{p_{1}(t)}\right]=\frac{H_{1}(t)}{p_{1}(t)}+\frac{H_{2}(t) e}{p_{1}(t)},
$$

where $H_{1}(t)$ is the nominal supply of currency 1 at time $t$, and $H_{2}(t)$ is the nominal supply of currency 2 at time $t$. The supply of each currency is kept constant and thus $H_{1}(t)=H_{1}(0)=H_{1}$, and $H_{2}(t)=H_{2}(0)=H_{2}$ for all $t$. Note that in this economy, the real money demands for individual currencies are not 
well defined. ${ }^{3}$ As a consequence, the exchange rate between the two currencies is indeterminate.

The indeterminacy of exchange rate proposition (Kareken and Wallace, 1981) states that if there exists a monetary equilibrium in which both currencies are valued at some exchange rate $e$, then there exists a monetary equilibrium at any exchange rate $e \in(0, \infty)$. Consider an exchange rate $\hat{e}, \hat{e} \neq e$, and the price sequences $\left\{\hat{p}_{1}(t)\right\}$ and $\left\{\hat{p}_{2}(t)\right\}, \hat{p}_{1}(t) \neq p_{1}(t)$ and $\hat{p}_{2}(t) \neq p_{2}(t)$ for $t \geq 1$ such that

$$
\hat{p}_{1}(t)=\frac{\left(H_{1}+\hat{e} H_{2}\right) p_{1}(t)}{H_{1}+e H_{2}}, \quad \hat{p}_{2}(t)=\hat{p}_{1}(t) / \hat{e} .
$$

The price sequences defined in Eq. (6) result in the same sequence of real rates of return as the price sequences $\left\{p_{1}(t)\right\}$ and $\left\{p_{2}(t)\right\}$ and, in turn, in the same values of individual and aggregate savings. Solving (6) for $\hat{p}_{1}(t)$ and substituting into (5) gives the following equilibrium condition:

$$
S(t)=\frac{H_{1}+\hat{e} H_{2}}{\hat{p}_{1}(t)} .
$$

Price levels $\hat{p}_{1}(t)$ and $\hat{p}_{2}(t)$ adjust enough to achieve identical values of savings in a monetary equilibrium with the exchange rate $e$ and in a monetary equilibrium with the exchange rate $\hat{e}$. Except for the initially old, who experience different consumption allocations for different initial nominal price levels, all other generations face the same consumption allocations in the equilibrium with the exchange rate $e$ as they do in the equilibrium with the exchange rate $\hat{e}$.

For any given exchange rate $e, e \in(0, \infty)$, this economy with constant supplies of both currencies has a stationary equilibrium in which the price level $p_{1}(t)$ (and $\left.p_{2}(t)\right)$ is constant. Notice that from Eq. (5) we can obtain the expression for the nominal price level $p_{1}(t)$ given by

$$
p_{1}(t+1)=\frac{w^{1}}{w^{2}} p_{1}(t)-\frac{H_{1}+e H_{2}}{N w^{2}} .
$$

In a stationary equilibrium, $p_{1}(t+1)=p_{1}(t)=p_{1}^{*}$ and

$$
p_{1}^{*}=\frac{H_{w}}{N\left(w^{1}-w^{2}\right)},
$$

where $H_{w}=H_{1}+e H_{2}$ is the world money supply. The level of $p_{1}^{*}$ depends on the value of the exchange rate $e$. In a stationary equilibrium, consumption allocations $c^{1, *}=c_{t}(t)$ and $c^{2, *}=c_{t}(t+1)$ are Pareto optimal with $c^{1, *}=c^{2, *}$ for all $t$.

\footnotetext{
${ }^{3}$ If there were a restriction that residents of each country could use only their country's currencies, real money demands for currencies 1 and 2 would be well defined and equal to the respective real money supplies of the two currencies.
} 


\subsection{Genetic algorithm}

At each time period $t \geq 1$, there are two populations of chromosomes, one being the new population of generation $t$, the young, the other being the population of generation $t-1$, the old. A population of generation $t$ consists of $N$ chromosomes that represent decision rules about first-period consumption and portfolio fraction for $N$ agents. A chromosome is a string of finite length $\ell$, written over the binary alphabet $\{0,1\}$.

A binary string $i, i \in[1, N]$, of length $l=30$ encodes the savings and the portfolio decision that an agent takes when young. The first 20 elements of a string encode the first-period consumption, $c_{i, t}(t)$, of agent $i$ of generation $t$. The remaining 10 elements encode the portfolio fraction of agent $i, f_{i}(t)$, which determines agent $i$ 's real money holdings of currency 1 and real money holdings of currency 2 at time $t$. For a string $i$ of length 30 the decoding is performed in the following way:

$$
x_{i, t}^{c}=\sum_{k=1}^{20} a_{i, t}^{k} 2^{k-1}, \quad x_{i, t}^{f}=\sum_{k=21}^{30} a_{i, t}^{k} 2^{k-21},
$$

where $a_{i, t}^{k}$ is the value $(0,1)$ taken at the $k$ th position in the $i$ th string. An integer number $x_{i, t}^{c}$ is obtained by decoding the consumption part of a binary string $i$ of generation $t$ and an integer number $x_{i, t}^{f}$ is obtained by decoding the fraction part of a string $i$ of generation $t$. These integer numbers are then normalized in order to obtain real number values, quantity $c_{i, t}(t) \in\left[0, w^{1}\right]$ that agent $i$ of generation $t$ decides to consume at time $t$ and fraction $f_{i}(t) \in[0,1]$ that is interpreted as agent $i$ 's portfolio decision:

$$
c_{i, t}(t)=\frac{x_{i, t}^{c}}{K^{c}}, \quad f_{i}(t)=\frac{x_{i, t}^{f}}{K^{f}},
$$

where $K^{c}=\left(2^{20}-1\right) / 10=104,857.5$ is a coefficient chosen to normalize the value of $x_{i, t}^{c}$ and $K^{f}=2^{10}-1=1,023$ is a coefficient chosen to normalize the value of $x_{i, t}^{f}$. The difference between $w^{1}$ and $c_{i, t}(t)$ gives the savings decision, $s_{i}(t)$, of agent $i$ at time $t$ :

$$
s_{i}(t)=w^{1}-c_{i, t}(t) .
$$

Agent $i$ places the fraction $f_{i}(t)$ of the savings $s_{i}(t)$ into currency 1 and the fraction $1-f_{i}(t)$ into currency 2. Aggregate savings, together with the holdings of currencies 1 and 2 of the members of generation $t-1$, determine the nominal price levels of the good that prevail at time $t$. The prices of the consumption good in terms of currency $1, p_{1}(t)$, and in terms of currency $2, p_{2}(t)$ are computed in the following way:

$$
p_{1}(t)=H_{1} / \sum_{i}^{N} f_{i}(t) s_{i}(t), \quad p_{2}(t)=H_{2} / \sum_{i}^{N}\left(1-f_{i}(t)\right) s_{i}(t) .
$$


The ratio of the nominal price levels yields the exchange rate $e(t)$. Given $p_{1}(t)$ and $p_{2}(t)$, the fraction $f_{i}(t)$ will determine the nominal holdings of currency 1 of agent $i, i \in[1, N]$, of generation $t$,

$$
m_{i, 1}(t)=f_{i}(t) s_{i}(t) p_{1}(t)
$$

and his nominal holdings of currency 2 ,

$$
m_{i, 2}(t)=\left(1-f_{i}(t)\right) s_{i}(t) p_{2}(t) .
$$

When old, agents use up all of their holdings of currencies 1 and 2 to purchase the consumption good. Thus the second-period consumption value of member of generation $t-1$ is given by

$$
c_{i, t-1}(t)=w^{2}+s_{i}(t-1)\left[f_{i}(t-1) \frac{p_{1}(t-1)}{p_{1}(t)}+\left(1-f_{i}(t-1)\right) \frac{p_{2}(t-1)}{p_{2}(t)}\right] .
$$

Finally, fitness values of the members of generation $t-1$ are computed. The fitness, $\mu_{i, t-1}$, of a string $i$ is given by the ex post value of the utility function of agent $i$ of generation $t-1$ :

$$
\mu_{i, t-1}=U\left(c_{i, t}(t), c_{i}(t+1)\right)=c_{i, t-1}(t-1) c_{i, t-1}(t) .
$$

New decision rules are generated through the application of the genetic algorithm operators: reproduction, crossover, mutation and election. The population of updated rules is then used by members of generation $t+1$.

Reproduction makes copies of individual strings. It promotes well-performing strings over time. The probability that a string will be copied is proportional to its fitness value. Thus a probability that a string $i$ of generation $t-1$ will get a copy $C_{i, t-1}$ is given by

$$
P\left(C_{i, t-1}\right)=\frac{\mu_{i, t-1}}{\sum_{i=1}^{N} \mu_{i, t-1}}, \quad i \in[1, N] .
$$

Reproduction operates like a biased roulette wheel. Each string is allocated a slot sized in proportion to its fitness. The number of spins of the wheel is equal to the number of chromosomes in a population and each spin yields a reproduction candidate. When a chromosome is selected, its exact copy is made. Once $N$ copies are made (the number of strings in a population is kept constant), they enter into a mating pool to undergo application of other genetic operators.

Crossover exchanges parts of randomly selected strings. First, two binary strings are selected from the mating pool at random. Secondly, an integer number $k$ is selected from $(1, \ldots, \ell-1)$, again at random. Two new strings are formed by swapping the set of values to the right of the position $k$. The total 
number of pairs that is selected is $N / 2$ (where $N$ is an even integer). Crossover takes place on each pair with probability pcross. An example of the crossover between two chromosomes for $\ell=10$ and $k=6$ is given below:

\section{0|1000 parent 1 \\ 000101|0110 parent 2.}

After the application of crossover, two resulting strings are:

1100100110 offspring 1

0001011000 offspring 2.

Two crossover operations are performed for each selected parent pair. One takes place on the parts of parent strings that decode the values of the firstperiod consumption. The other takes place on the parts of parent strings that decode the values of portfolio fractions.

Mutation randomly alters the value of a position within a string to the other value taken by the binary alphabet: $0 \rightarrow 1$ and $1 \rightarrow 0$. The probability of mutation, pmut, is independent and identical across the positions.

The election operator (Arifovic, 1994) tests newly generated offspring before they can enter into a population of generation $t+1$. It is applied in the following way. First, a binary string $i, i \in[1 \ldots N]$, that belongs to a new offspring $i$ is decoded into a real number that represents offspring $i$ 's first-period consumption decision, $c_{i, t+1}(t+1)$. Based on this decision and on the rate of return between periods $t-1$ and $t$, a second-period consumption value for offspring $i$ is computed. The values of $c_{i, t}(t)$ and $c_{i, t}(t+1)$ are then used to compute offspring i's potential fitness value, $\mu_{i}^{\mathrm{p}}(t+1)$.

Second, two offspring and two parents (recorded at the crossover and mutation stages) are ranked, from the highest to the lowest, on the basis of the fitness values. The two strings that have the first and the second rank are chosen and placed into the population of a new generation. In case that an offspring and a parent have the same fitness value, an offspring is placed into the population.

Thus a population of chromosomes that will represent decision rules of young agents at $t+1$ is generated in the following way: Firstly, the application of the reproduction operator to the population of rules of generation $t$ yields a population of $N$ copies. Secondly, crossover and mutation are applied to generate new decision rules to be tested. Finally, newly generated offspring are subjected to the election operator test. Offspring that pass the test, together with parents that are more fit than their offspring, form a new population of decision rules of agents born at time period $t+1$.

The genetic algorithm simulations resulted in the fluctuating patterns of the exchange rate that never settled down to a constant value. The stability analysis showed that a stationary perfect foresight monetary equilibrium of the Kareken-Wallace model was not stable under the genetic algorithm dynamics. 
In case that the genetic algorithm economy reaches a stationary equilibrium in which all agents use identical equilibrium rules, it will not remain there since the genetic algorithm agents adopt new rules with portfolio fraction values different from the equilibrium ones.

\section{Time series properties}

We conducted simulations and analyzed the generated data for two different sets of the overlapping-generations model parameter values: $w_{1}=10$ and $w_{2}=$ 4 , and $w_{1}=10$ and $w_{2}=1$. For both of these sets of the endowment patterns, the supplies of the currencies were given by: $H_{1}=3000$ and $H_{2}=3600$. Each of these two sets was combined with 8 different sets of the crossover and mutation rate values. Each combination of the overlapping-generations parameter values and the genetic algorithm parameter values was simulated for three different random number seeds. Thus we conducted the total of 48 simulations. Each simulation lasted for 10,000 time periods.

Our simulations were initialized randomly. Each bit at every position in a binary string had a $50 \%$ chance of being equal to 0 or to 1 . This implied that the average initial values of the first-period consumption were equal to $w^{1} / 2$ and the average initial values of the portfolio fraction was equal to 0.5 .

We analyze the statistical properties of the exchange rate returns. The exchange rate $e(t)=p_{1}(t) / p_{2}(t)$ is equal to the ratio of the nominal price levels in terms of currencies 1 and 2 . In the rest of the paper, we use exchange rate returns and this is calculated by $r(t)=\log (e(t) / e(t-1))$. The dynamics of the exchange rates for $w^{1}=10, w^{2}=4, H_{1}=3000, H_{2}=3600$, pcross $=0.6$ and pmut $=$ 0.033 are shown in Fig. 1. The dynamics for other combinations of the crossover and mutation rate parameters are similar in two sets of the overlappinggenerations models.

We analyze the exchange rate returns in three segments. These are the first 500 observations, the first 5000 observations and the last 5000 observations. In Table 1, the first 10 autocorrelations of the exchange rate returns for these three data segments are reported for $w^{1}=10, w^{2}=4, H_{1}=3000, H_{2}=3600$, pcross $=0.6$ and pmut $=0.033$. The Ljung-Box-Pierce test ${ }^{4}$ for the first 500 observations reject the null hypothesis of identical and independent observations at the 5\% level. For the first and the last 5000 observations, the persistence in these series is stronger and the Ljung-Box-Pierce test values are 879 and $0.108 \times 10^{4}$, respectively. The autocorrelations of the three data segments follow

\footnotetext{
${ }^{4}$ The Ljung-Box-Pierce statistic is distributed $\chi^{2}(10)$ under the null hypothesis of identical and independent observations.
} 


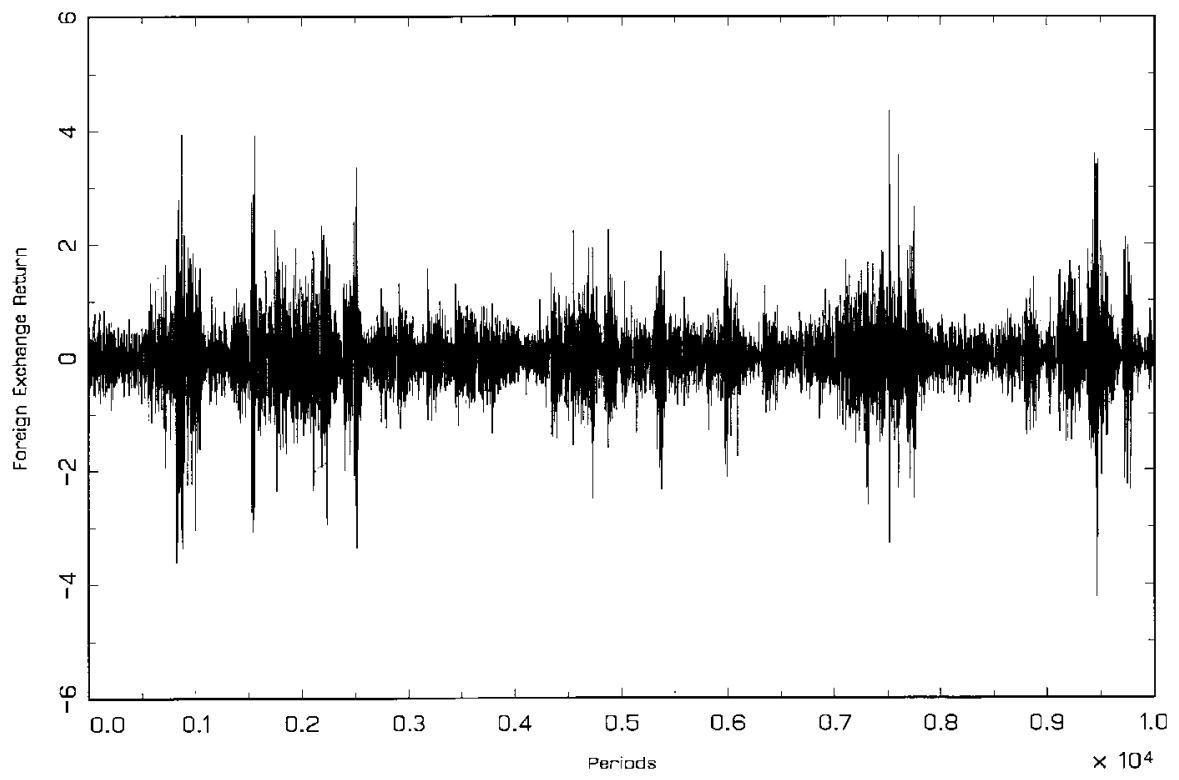

Fig. 1. Foreign exchange return dynamics.

Table 1

Autocorrelations of the foreign exchange returns

\begin{tabular}{lccr}
\hline Description & $\begin{array}{l}\text { First } 500 \\
\text { observations }\end{array}$ & $\begin{array}{l}\text { First 5000 } \\
\text { observations }\end{array}$ & $\begin{array}{l}\text { Last } 5000 \\
\text { observations }\end{array}$ \\
\hline$\rho_{1}$ & -0.13111 & 0.16960 & 0.20714 \\
$\rho_{2}$ & -0.08681 & -0.24955 & -0.21882 \\
$\rho_{3}$ & -0.09293 & -0.22266 & -0.24517 \\
$\rho_{4}$ & -0.18620 & -0.14192 & -0.21861 \\
$\rho_{5}$ & -0.09855 & -0.07921 & -0.10633 \\
$\rho_{6}$ & 0.02401 & -0.02401 & 0.03396 \\
$\rho_{7}$ & -0.08119 & 0.01858 & 0.04312 \\
$\rho_{8}$ & -0.06479 & 0.05991 & 0.05612 \\
$\rho_{9}$ & 0.01362 & 0.04776 & 0.00842 \\
$\rho_{10}$ & 0.06838 & 0.04274 & -0.00241 \\
Bartlett std. errors & 0.0447 & 0.0141 & 0.0141 \\
LBP & 47.6 & 879.0 & $0.108 \times 10^{4}$ \\
$\chi_{0.05}^{2}(10)$ & 18.307 & & \\
\hline
\end{tabular}

Notes: $\rho_{1}, \ldots, \rho_{10}$ are the first 10 autocorrelations of each series. LBP refers to the Ljung-Box-Pierce statistic and it is distributed $\chi^{2}(10)$ under the null hypothesis of identical and independent observations. 
Table 2

BDS tests and the Lyapunov exponents for the foreign exchange returns $w^{1}=10, w^{2}=4$, $H_{1}=3000, H_{2}=3600$, pcross $=0.6$, pmut $=0.033$

\begin{tabular}{llll}
\hline Description & $\begin{array}{l}\text { First } 500 \\
\text { observations }\end{array}$ & $\begin{array}{l}\text { First } 5000 \\
\text { observations }\end{array}$ & $\begin{array}{l}\text { Last } 5000 \\
\text { observations }\end{array}$ \\
\hline
\end{tabular}

$\begin{array}{crrr}\text { BDS }(\varepsilon=0.75 \sigma) & & & \\ m=2 & 6.09 & 19.28 & 24.10 \\ m=3 & 8.80 & 32.81 & 37.36 \\ m=4 & 8.18 & 40.12 & 45.84 \\ m=5 & 9.66 & 51.46 & 57.03 \\ \text { BDS }(\varepsilon=0.50 \sigma) & & & \\ m=2 & 14.09 & 26.45 & 27.02 \\ m=3 & 18.70 & 43.38 & 43.47 \\ m=4 & 20.42 & 54.98 & 56.41 \\ m=5 & 25.78 & 75.16 & \\ \operatorname{BDS}(\varepsilon=0.25 \sigma) & & & \\ m=2 & 21.96 & 44.15 & 37.88 \\ m=3 & 29.25 & 72.53 & 63.48 \\ m=4 & 35.57 & 104.24 & 150.01 \\ m=5 & 46.58 & 165.85 & \end{array}$

Lyapunov exponents

$\begin{array}{rrrr}\lambda_{1} & 0.0331 & 0.1218 & 0.1773 \\ \lambda_{2} & -1.0832 & -0.1027 & -0.1221\end{array}$

Notes: $\sigma$ is the standard deviation of the exchange rates. $\sigma$ is $0.29950,0.60031$ and 0.54968 for the exchange rates in the first, second and the third columns, respectively. $m$ refers to the embedding dimension. $\varepsilon$ is the distance parameter and is chosen to be the fraction of the standard deviation of the data. BDS test is distributed standard normal asymptotically. $\lambda_{1}$ and $\lambda_{2}$ refer to the largest two Lyapunov exponents.

similar patterns. The autocorrelations for other combinations of the crossover and mutation rate parameters are similar in two sets of the overlappinggenerations models and are not reported here.

In addition, we calculated the BDS statistic of Brock et al. (1996). ${ }^{5}$ The BDS statistic tests the null hypothesis of identical and independent distribution and it is shown to have good power against nonlinear alternatives. It is distributed asymptotically standard normal. The calculations are reported in Tables 2-5 for two different sets of crossover and mutation values of the two overlappinggenerations models. ${ }^{6}$ The BDS analysis is carried out in three segments of each

\footnotetext{
${ }^{5}$ The BDS test is calculated by the LeBaron (1997) routine.

${ }^{6}$ The remaining six different sets of crossover and mutation values for two sets of overlappinggenerations models are similar to the ones presented in Tables 2-5 and are not reported here.
} 
Table 3

BDS tests and the Lyapunov exponents for the foreign exchange returns $w^{1}=10, w^{2}=4$, $H_{1}=3000, H_{2}=3600$, pcross $=0.75$, pmut $=0.033$

\begin{tabular}{lccc}
\hline Description & $\begin{array}{c}\text { First } 500 \\
\text { observations }\end{array}$ & $\begin{array}{l}\text { First } 5000 \\
\text { observations }\end{array}$ & $\begin{array}{c}\text { Last } 5000 \\
\text { observations }\end{array}$ \\
\hline BDS $(\varepsilon=0.75 \sigma)$ & & & \\
$m=2$ & 5.02 & 24.50 & 25.31 \\
$m=3$ & 11.52 & 41.88 & 42.31 \\
$m=4$ & 13.32 & 54.93 & 55.16 \\
$m=5$ & 16.75 & 72.55 & 73.86 \\
BDS $(\varepsilon=0.50 \sigma)$ & & & \\
$m=2$ & 8.03 & 26.29 & 29.22 \\
$m=3$ & 16.01 & 48.56 & 76.40 \\
$m=4$ & 19.55 & 71.16 & 119.24 \\
$m=5$ & 26.16 & 106.84 & \\
BDS $(\varepsilon=0.25 \sigma)$ & & & 37.30 \\
$m=2$ & 13.79 & 33.03 & 73.09 \\
$m=3$ & 24.86 & 67.04 & 132.51 \\
$m=4$ & 32.70 & 115.63 & 269.79 \\
$m=5$ & 47.76 & 219.33 & \\
Lyapunov exponents & & & 0.1474 \\
$\lambda_{1}$ & 0.1471 & 0.1713 & -1.2701 \\
$\lambda_{2}$ & -0.8921 & -0.9818 & \\
\hline
\end{tabular}

Notes: $\sigma$ is the standard deviation of the exchange rates. $\sigma$ is $0.6535,0.5927$ and 0.5645 for the exchange rates in the first, second and the third columns, respectively. $m$ refers to the embedding dimension. $\varepsilon$ is the distance parameter and is chosen to be the fraction of the standard deviation of the data. BDS test is distributed standard normal asymptotically. $\lambda_{1}$ and $\lambda_{2}$ refer to the largest two Lyapunov exponents.

data set. For all three segments of each exchange rate series, the BDS statistic rejects the null hypothesis of identical and independent distribution in all series except a few cases. Therefore, the Ljung-Box-Pierce statistic and the BDS statistic results clearly rule out the lack of persistence in the foreign exchange series.

To investigate the sources of this persistence, the phase diagrams in Figs. 2 and 3 are plotted. Figs. 2(a) and (b) provide the phase diagrams for the last 5000 observations of the exchange rates for the first parameter set of the overlappinggenerations model and two combinations of the crossover and mutation rates. Figs. 3(a) and (b) are for the second parameter set of the overlapping-generations model and for the two combinations of the crossover and mutation rates. The interesting aspects of the figures are that there is a well-defined attractor for the 
Table 4

BDS tests and the Lyapunov exponents for the foreign exchange returns $w^{1}=10, w^{2}=1, H_{1}=3000, H_{2}=3600$, pcross $=0.6$, pmut $=0.033$

\begin{tabular}{lccr}
\hline Description & $\begin{array}{l}\text { First } 500 \\
\text { observations }\end{array}$ & $\begin{array}{l}\text { First } 5000 \\
\text { observations }\end{array}$ & $\begin{array}{l}\text { Last } 5000 \\
\text { observations }\end{array}$ \\
\hline BDS $(\varepsilon=0.75 \sigma)$ & & & \\
$m=2$ & -1.71 & -0.94 & 1.21 \\
$m=3$ & 5.08 & 23.04 & 27.54 \\
$m=4$ & 6.60 & 28.60 & 44.03 \\
$m=5$ & 9.83 & 42.84 & 70.65 \\
BDS $(\varepsilon=0.50 \sigma)$ & & & \\
$m=2$ & 0.93 & 7.76 & 11.30 \\
$m=3$ & 9.88 & 37.62 & 47.25 \\
$m=4$ & 14.38 & 51.54 & 86.92 \\
$m=5$ & 22.19 & 83.01 & 164.59 \\
BDS $(\varepsilon=0.25 \sigma)$ & & & 32.62 \\
$m=2$ & 5.03 & 22.06 & 104.55 \\
$m=3$ & 16.96 & 61.74 & 266.55 \\
$m=4$ & 29.14 & 102.70 & 746.88 \\
$m=5$ & 56.85 & 205.75 & \\
Lyapunov exponents & & & 0.2971 \\
$\lambda_{1}$ & 0.0410 & 0.2611 & -1.0048 \\
$\lambda_{2}$ & -0.9631 & -1.1211 & \\
\hline
\end{tabular}

Notes: $\sigma$ is the standard deviation of the exchange rates. $\sigma$ is $1.7760,1.4226$ and 1.2446 for the exchange rates in the first, second and the third columns, respectively. $m$ refers to the embedding dimension. $\varepsilon$ is the distance parameter and is chosen to be the fraction of the standard deviation of the data. BDS test is distributed standard normal asymptotically. $\lambda_{1}$ and $\lambda_{2}$ refer to the largest two Lyapunov exponents.

foreign exchange rates for all series and the shape of the attractor is robust to the changes in the overlapping-generations model parameters as well as in the changes for the crossover and mutation rates.

To verify whether this attractor is chaotic, the largest two Lyapunov exponents are calculated and reported in Tables 2-5. The Lyapunov exponents are estimated by the Gençay and Dechert (1992) algorithm ${ }^{7}$ which is a Jacobianbased Lyapunov exponent algorithm and shown to have good small sample properties. If the attractor under investigation is dissipative, the positive Lyapunov exponent is a sufficient condition for the existence of chaotic attractor. The largest Lyapunov exponent $\left(\lambda_{1}\right)$ is positive in all segments of each data

\footnotetext{
${ }^{7}$ An application of this algorithm with the spot exchange rates is presented in Dechert and Gençay (1992).
} 
Table 5

BDS tests and the Lyapunov exponents for the foreign exchange returns $w^{1}=10, w^{2}=1$, $H_{1}=3000, H_{2}=3600$, pcross $=0.75$, pmut $=0.033$

\begin{tabular}{llll}
\hline Description & $\begin{array}{l}\text { First } 500 \\
\text { observations }\end{array}$ & $\begin{array}{l}\text { First } 5000 \\
\text { observations }\end{array}$ & $\begin{array}{l}\text { Last } 5000 \\
\text { observations }\end{array}$ \\
\hline
\end{tabular}

$\begin{array}{crrr}\operatorname{BDS}(\varepsilon=0.75 \sigma) & & & \\ m=2 & -0.80 & -2.89 & 0.50 \\ m=3 & 6.74 & 23.50 & 24.33 \\ m=4 & 8.27 & 35.61 & 38.96 \\ m=5 & 12.15 & 58.42 & 64.36\end{array}$

$\operatorname{BDS}(\varepsilon=0.50 \sigma)$

$\begin{array}{lr}m=2 & 1.29 \\ m=3 & 10.26 \\ m=4 & 14.7 \\ m=5 & 22.87\end{array}$

$\begin{array}{ll}116.74 & 150.20\end{array}$

$\begin{array}{crrr}\operatorname{BDS}(\varepsilon=0.25 \sigma) & & & \\ m=2 & 5.67 & 17.54 & 27.18 \\ m=3 & 16.78 & 71.91 & 89.26 \\ m=4 & 28.99 & 168.20 & 220.71 \\ m=5 & 57.12 & 467.16 & 605.68\end{array}$

Lyapunov exponents
$\lambda_{1}$
0.0464
0.1512
0.2226
$\lambda_{2}$
$-0.8244$
$-0.8711$
$-0.9779$

Notes: $\sigma$ is the standard deviation of the exchange rates. $\sigma$ is $1.4636,1.3349$ and 1.3986 for the exchange rates in the first, second and the third columns, respectively. $m$ refers to the embedding dimension. $\varepsilon$ is the distance parameter and is chosen to be the fraction of the standard deviation of the data. BDS test is distributed standard normal asymptotically. $\lambda_{1}$ and $\lambda_{2}$ refer to the largest two Lyapunov exponents.

set and it is robust to changes in the overlapping-generations model and the genetic algorithm parameters. The positiveness of the largest Lyapunov exponents in Tables 2-5 confirm that attractors under investigation are chaotic.

As a further check, the linear structure in the foreign exchange returns is pre-filtered and the BDS test is calculated from the residuals. The autoregressive (AR) model with 10 lags, AR(10), has sufficiently large number of lags to capture the linear persistence in the series. ${ }^{8}$ In addition to the AR(10) model, the $\operatorname{AR}(10)-G A R C H(1,1)$ model is also estimated and the normalized residuals are tested with the BDS test. The $\operatorname{AR}(10)-\operatorname{GARCH}(1,1)$ model is estimated to pre-filter any nonlinearities originating from the potential volatility clustering.

\footnotetext{
${ }^{8}$ The AR(10) model improves on the AR(5) model based on information theoretic criteria.
} 

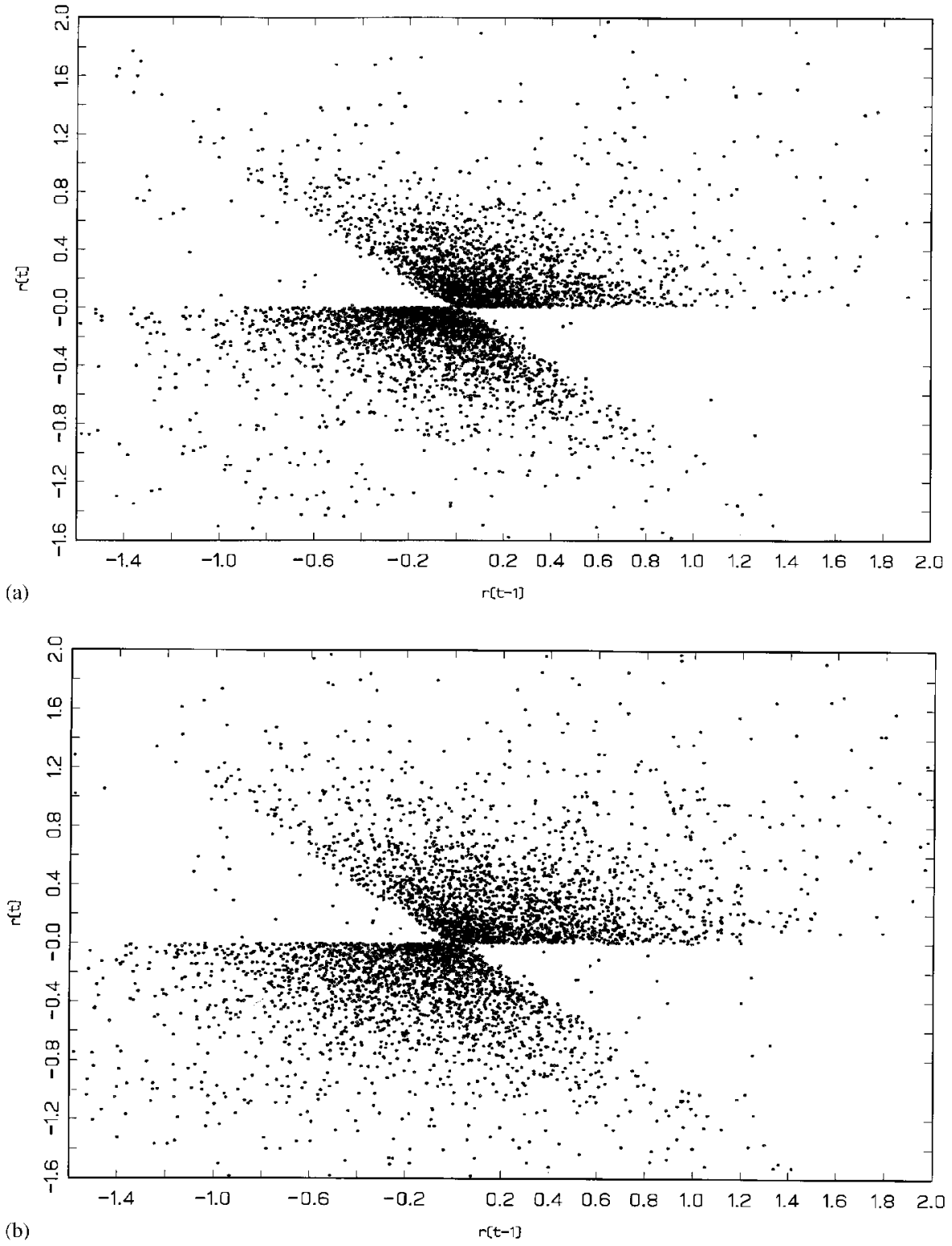

Fig. 2. (a) Phase portrait of foreign exchange returns for OLG Model I: pcross $=0.6$, pmut $=0.033$.

(b) Phase portrait of foreign exchange returns for OLG Model I: pcross $=0.75$, pmut $=0.033$. 

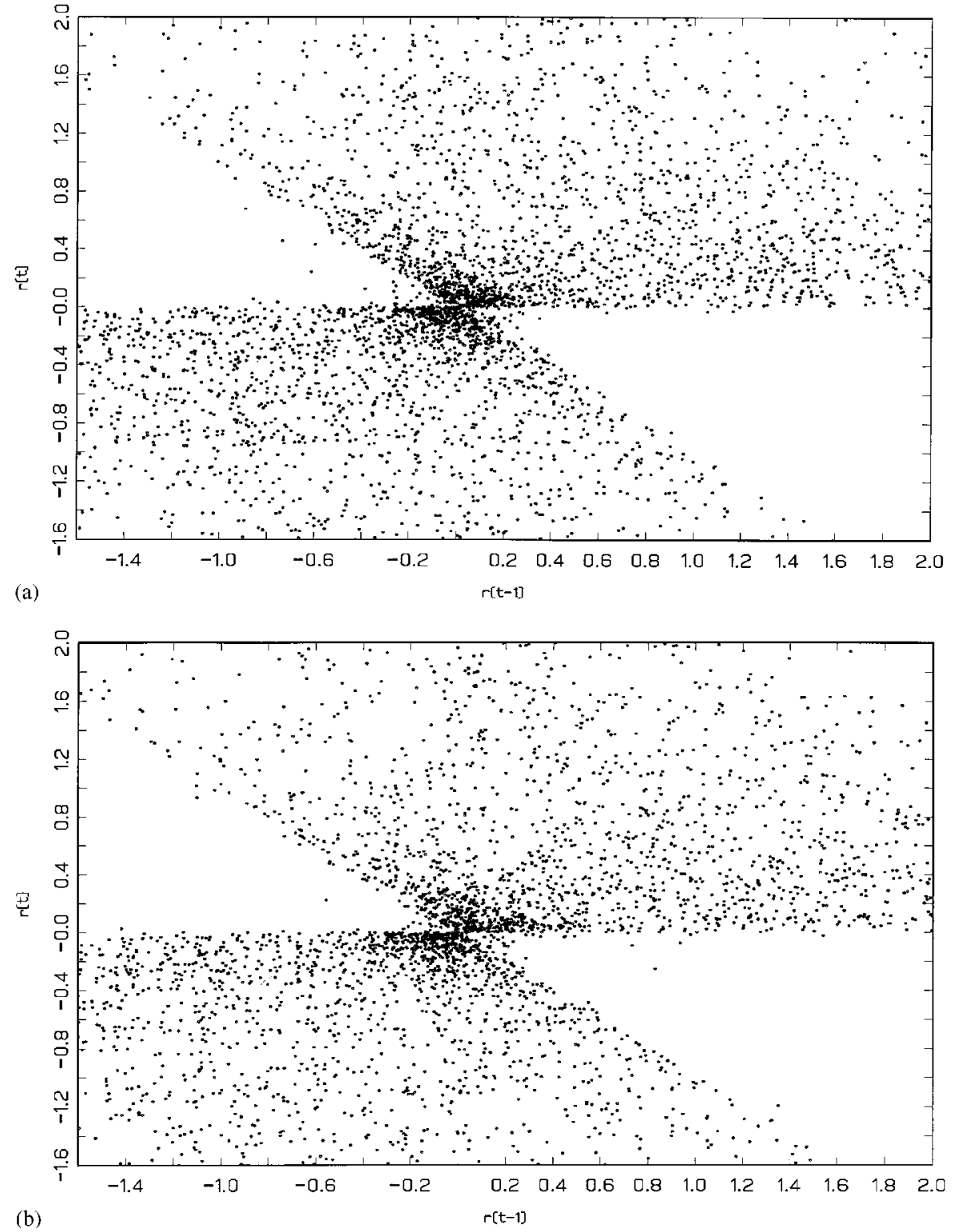

Fig. 3. (a) Phase portrait of foreign exchange returns for OLG Model II: $p c r o s s=0.6, p m u t=0.033$.

(b) Phase portrait of foreign exchange returns for OLG Model II: pcross $=0.75$, pmut $=0.033$. 
Table 6

BDS tests from the $\mathrm{AR}(10)$ and $\mathrm{AR}(10)-\mathrm{GARCH}(1,1)$ residuals OLG Model I

\begin{tabular}{|c|c|c|c|c|}
\hline \multirow[t]{2}{*}{ Description } & \multicolumn{2}{|c|}{ pcross $=0.60$, pmut $=0.033$} & \multicolumn{2}{|c|}{ pcross $=0.75$, pmut $=0.033$} \\
\hline & $\operatorname{AR}(10)$ & $\operatorname{AR}(10)-G A R C H(1,1)$ & $\operatorname{AR}(10)$ & $\operatorname{AR}(10)-\operatorname{GARCH}(1,1)$ \\
\hline \multicolumn{5}{|c|}{$\operatorname{BDS}(\varepsilon=0.75 \sigma)$} \\
\hline$m=2$ & 25.12 & 0.34 & 24.03 & 3.14 \\
\hline$m=3$ & 34.13 & 2.90 & 33.85 & 5.11 \\
\hline$m=4$ & 43.06 & 5.91 & 45.95 & 7.73 \\
\hline$m=5$ & 55.46 & 9.91 & 62.71 & 11.51 \\
\hline \multicolumn{5}{|c|}{$\operatorname{BDS}(\varepsilon=0.50 \sigma)$} \\
\hline$m=2$ & 25.95 & 4.55 & 26.42 & 7.12 \\
\hline$m=3$ & 36.61 & 7.70 & 40.54 & 10.16 \\
\hline$m=4$ & 50.74 & 14.23 & 63.86 & 16.26 \\
\hline$m=5$ & 72.30 & 21.58 & 101.94 & 23.76 \\
\hline \multicolumn{5}{|c|}{$\operatorname{BDS}(\varepsilon=0.25 \sigma)$} \\
\hline$m=2$ & 27.22 & 8.14 & 29.14 & 10.30 \\
\hline$m=3$ & 40.62 & 12.88 & 50.05 & 15.52 \\
\hline$m=4$ & 65.46 & 22.85 & 98.28 & 26.54 \\
\hline$m=5$ & 111.54 & 35.47 & 204.55 & 40.54 \\
\hline
\end{tabular}

Notes: $\sigma$ is the standard deviation of normalized residuals. $m$ refers to the embedding dimension. $\varepsilon$ is the distance parameter and is chosen to be the fraction of the standard deviation of the normalized residuals.

The results are presented in Tables 6 and 7 for the two overlapping generations economies, respectively. The results indicate that there is a substantial amount of persistence left over in the residuals of the AR(10) model as the BDS test rejects the null hypothesis of the identical and independent distribution of the $\mathrm{AR}(10)$ residuals. When evaluated with the BDS test, the degree of persistence in the AR(10)-GARCH $(1,1)$ residuals are much smaller but still statistically significant for both overlapping generations models. Therefore, this further check with the pre-filtered data indicate that the persistence in the foreign exchange returns cannot be captured by a linear AR model or an AR model which accounts for volatility clustering.

\section{The analysis of the dynamics}

The exhibited dynamics are robust in regard to the changes in the parameter values of both the economic model and the genetic algorithm. The observed persistence in the simulated data is due to the joint effects related to the indeterminacy of equilibria and the dynamics generated by the genetic algorithm operators. Arifovic (1996) demonstrates that a stationary equilibrium of 
Table 7

BDS tests from the $\mathrm{AR}(10)$ and $\mathrm{AR}(10)-\mathrm{GARCH}(1,1)$ residuals OLG Model II

\begin{tabular}{|c|c|c|c|c|}
\hline \multirow[t]{2}{*}{ Description } & \multicolumn{2}{|c|}{ pcross $=0.60$, pmut $=0.033$} & \multicolumn{2}{|c|}{ pcross $=0.75$, pmut $=0.033$} \\
\hline & $\operatorname{AR}(10)$ & AR(10)-GARCH(1,1) & $\operatorname{AR}(10)$ & $\operatorname{AR}(10)-G A R C H(1,1)$ \\
\hline \multicolumn{5}{|c|}{$\operatorname{BDS}(\varepsilon=0.75 \sigma)$} \\
\hline$m=2$ & 14.48 & -5.56 & 10.84 & -1.83 \\
\hline$m=3$ & 47.21 & 4.13 & 40.29 & 7.55 \\
\hline$m=4$ & 72.83 & 8.49 & 67.59 & 11.89 \\
\hline$m=5$ & 125.58 & 16.64 & 112.26 & 19.77 \\
\hline \multicolumn{5}{|c|}{$\operatorname{BDS}(\varepsilon=0.50 \sigma)$} \\
\hline$m=2$ & 23.43 & -2.89 & 22.11 & 3.20 \\
\hline$m=3$ & 72.00 & 8.71 & 69.99 & 14.49 \\
\hline$m=4$ & 142.86 & 17.22 & 142.33 & 23.01 \\
\hline$m=5$ & 287.71 & 31.47 & 293.49 & 37.47 \\
\hline \multicolumn{5}{|c|}{$\operatorname{BDS}(\varepsilon=0.25 \sigma)$} \\
\hline$m=2$ & 42.87 & -0.29 & 40.94 & 6.79 \\
\hline$m=3$ & 136.87 & 13.57 & 137.34 & 21.55 \\
\hline$m=4$ & 392.07 & 25.76 & 389.79 & 35.82 \\
\hline$m=5$ & 1210.58 & 46.80 & 1218.14 & 64.71 \\
\hline
\end{tabular}

Notes: $\sigma$ is the standard deviation of normalized residuals. $m$ refers to the embedding dimension. $\varepsilon$ is the distance parameter and is chosen to be the fraction of the standard deviation of the normalized residuals.

this model in which both currencies are valued is unstable under the genetic algorithm dynamics.

Suppose that the genetic algorithm economy has been in a stationary equilibrium in $t-1$ and $t$. In a stationary equilibrium, all strings decode to the same values of the first-period consumption and portfolio fraction and the rates of return on two currencies are equal. When the genetic algorithm is applied at time $t+1$, reproduction and crossover have no effect. Mutation will bring diversity into the population, but whether the new strings, different from the equilibrium ones, become members of the actual populations depends on the election operator. This operator will admit all those strings whose portfolio fractions decode to numbers different from the stationary equilibrium ones, but that still have stationary equilibrium values of the first-period consumption. They pass the election operator test because their fitness is evaluated at the previous period rates of return on two currencies. Because the economy was in a stationary equilibrium in the previous two periods, the two rates of return were equal. This is the reason why the offspring with stationary values of the first-period consumption and portfolio fractions different from the stationary ones will have potential fitness value equal to the fitness value of their parents'. 
From the standpoint of the election operator, actual fractions placed in each currency do not matter since the rates of return were equal. Thus, the fitness value of these offspring will be the same as the fitness value of their parents' that decode to the stationary equilibrium values.

Once the diversity is brought into the populations, the rates of return on two currencies will no longer be equal. Further adaptation will favor strings that place higher fractions of savings into the currency with a higher rate of return. Consequently, even if the economy reaches a stationary equilibrium by chance or if it is initialized at a stationary equilibrium, the genetic algorithm dynamics will take it away from that stationary equilibrium.

In general, the out-of-equilibrium heterogeneity of the portfolio fraction values results in the inequality of the rates of return on two currencies. The genetic algorithm agents seek to exploit this arbitrage opportunity by placing larger fractions of their savings into the currency that had a higher rate of return in the previous period. If the aggregate change of the portfolio fraction is large enough, the direction of the inequality is preserved and the value of the currency with the higher rate of return increases. On the other hand, if the aggregate change is not large enough, the reversal of the inequality of the rates of return occurs. The reversal will prompt the genetic algorithm agents to place more savings into the currency whose value was decreasing prior to the reversal. As a result, the exchange rate changes the direction of movement. These dynamics bring about the fluctuations in the portfolio fraction and the exchange rate that persist over time.

While the set of equilibria indexed by the exchange rates in the range $(0, \infty)$ is unbounded, our analysis will focus on the bounded set of equilibria in which both currencies are valued. The value of $e(t)=0$ implies the equilibrium in which only currency 1 is valued, and the value of $e(t) \rightarrow \infty$ implies the equilibrium in which only currency 2 is valued. While these two equilibria are not a priori ruled out in the algorithm, we never observed them in the simulations, i.e. the genetic algorithm economies never reached the point where only one of the two currencies is used. ${ }^{9}$

\footnotetext{
${ }^{9}$ A version of the cash-in-advance model with three types of agents who face different portfolio restrictions (see King et al. (1992)) could be used in order to get a bounded set of values of the equilibrium exchange rate. In this model, type 1 can hold only currency 1 , type 2 only currency 2 , while type 3 has no restrictions on the portfolio holdings, i.e. type 3 can hold both currencies. The exchange rate is still indeterminate, but the range of values is bounded and the exchange rate is positive and finite for all possible values of the portfolio of type 3 . If type 3 agents were endowed with the genetic algorithm in order to decide on their portfolio, the exchange rate would exhibit similar dynamics. Again, a perfect foresight equilibrium in this economy would not be stable under the genetic algorithm dynamics, and the model would exhibit persistent fluctuations of the exchange rate. The source of instability would be the same as the one described above for our overlappinggenerations model.
} 
For the purpose of the subsequent analysis, we will assume that there is a lower bound and an upper bound on the average portfolio fraction, $\bar{f}(t)$, such that $\varepsilon<\bar{f}(t)<1-\varepsilon$ for all $t$, where $\varepsilon$ is arbitrarily small positive value. If $\bar{f}(t)=0$, currency 1 is not valued and if $\bar{f}(t)=1$, currency 2 is not valued. Thus this assumption guarantees that both currencies will always be valued, i.e. that both $p_{1}(t)$ and $p_{2}(t)$ always take strictly positive, finite values. In the implementation of the genetic algorithm, the value of $\varepsilon$ depends on the length of a part of a binary string used to decode the portfolio fraction. The smallest positive number between 0 and 1 that can be encoded is equal to $1 / K^{f}$ where $K^{f}=2^{l}-1$ and $l$ is the number of bits used to encode the portfolio fraction. Thus the larger the length, $l$, the smaller positive real number that can be encoded. In our simulations $l=10$ so that $K^{f}=1023$. In order to satisfy the lower bound on $\bar{f}(t), N-1$ agents can have $f_{i}(t)$ 's equal to 0 and only one agent with $f_{i, t}=1 / 1023$ is required. Likewise, to satisfy the upper bound, $N-1$ agents can have $f_{i}(t)$ 's equal to 1 and only one agent with $f_{i}(t)=1-1 / 1023 N$ is required. Thus the smallest value that $\varepsilon$ can take is $1 / 1023 N$.

The second assumption is related to the genetic algorithm value of the first-period consumption which is set equal to the stationary equilibrium value. This assumption is made in order to make the analysis tractable and, in fact, it captures the main feature of the behavior of the first-period consumption values that, after initial adjustment, remain very close to the stationary values (see Arifovic, 1996). Then, the rates of return on two currencies are given by

$$
\begin{aligned}
R_{1}(t) & =\frac{\sum_{i} f_{i}(t) s_{i}(t)}{\sum_{i} f_{i}(t-1) s_{i}(t-1)}=\frac{\bar{f}(t) s^{*}}{\bar{f}(t-1) s^{*}}, \\
R_{1}(t) & =\frac{\bar{f}(t)}{\bar{f}(t-1)}, \\
R_{2}(t) & =\frac{\sum_{i}\left(1-f_{i}(t)\right) s_{i}(t)}{\sum_{i}\left(1-f_{i}(t-1)\right) s_{i}(t-1)}=\frac{(1-\bar{f}(t)) s^{*}}{(1-\bar{f}(t-1)) s^{*}}, \\
R_{2}(t) & =\frac{(1-\bar{f}(t))}{(1-\bar{f}(t-1))} .
\end{aligned}
$$

Notice that $e(t) / e(t-1)=R_{2}(t) / R_{1}(t)$ and thus $r(t)=\log \left(R_{2}(t) / R_{1}(t)\right)=$ $\log R_{2}(t)-\log R_{1}(t)$. Let $h(t+1)$ define the ratio between the exchange rate returns at $t+1$ and $t, r(t+1)$ and $r(t)$. Then $h(t+1)$ is given by

$$
\frac{\log R_{2}(t+1)-\log R_{1}(t+1)}{\log R_{2}(t)-\log R_{1}(t)} .
$$

The values of this ratio depend on the values taken by the average portfolio fraction. When the absolute value of this ratio is greater than one, the dynamical 
system is expanding and when it is less than one, it is contracting. As noted earlier, the inequality of the rates of return on the two currencies is driving the changes in the portfolio fractions. For example, suppose that $R_{1}(t)>R_{2}(t) .{ }^{10} \mathrm{As}$ long as this direction of inequality is preserved, the genetic operators will work on increasing $\bar{f}(t)$, i.e. crossover and mutation will create strings with increasing values of $f_{i}(t)$ 's that the election operator will admit into the actual population of decision rules. The preservation of the direction of inequality requires the sequence of increasing values of $\bar{f}(t)$, i.e. $\bar{f}(t)>\bar{f}(t-1)$ for time periods during which $R_{1}(t)>R_{2}(t)$.

Notice that the probability of obtaining larger values of $\bar{f}(t)$ is decreasing in the size of $\bar{f}(t)$. In other words, as $\bar{f}(t)$ gets larger, the number of bits that can be changed in order to increase its value gets smaller, and the probability that exactly those bits will be changed gets smaller. (The same is true of the process that works in the opposite direction where the values of $\bar{f}(t)$ are decreasing since $R_{1}(t)<R_{2}(t)$.) If mutation does not bring about sufficient amount of change to make $\bar{f}(t)>\bar{f}(t-1)$, the direction of inequality is reversed, i.e. $R_{1}(t)<R_{2}(t)$, and the genetic algorithm starts working in the opposite direction, i.e. accepting strings with lower values of $f_{i}(t+1)$.

At time $t$, there are three possibilities for the relationship between the two rates of return, $R_{1}(t)>R_{2}(t), R_{1}(t)<R_{2}(t)$, and $R_{1}(t)=R_{2}(t)$. We will discuss the first two since the equality of the rates of return was never observed in our simulations. ${ }^{11}$ The direction of inequality at time $t$ together with the direction of inequality at time $t+1$ will determine the value of $h(t+1)$.

Consider first the case where $R_{1}(t)>R_{2}(t)$. At time $t+1$, the population of generation $t-1$ is contributing copies and offspring rules to be included into the population of generation $t+1$. Because $R_{1}(t)>R_{2}(t)$, the result of the election operator test will be that $\bar{f}(t+1) \geq \bar{f}(t-1)$. The relationship between $\bar{f}(t+1)$ and $\bar{f}(t)$ will determine the direction of inequality of the rates of return at time $t+1$. There are two possible outcomes: that $\bar{f}(t+1)>\bar{f}(t)$ which implies $R_{1}(t+1)>R_{2}(t+1)$, and that $\bar{f}(t+1)<\bar{f}(t)$ which implies $R_{1}(t+1)<$ $R_{2}(t+1)$.

If mutation results in an increase in the average portfolio fraction sufficient to preserve the direction of inequality, i.e. the outcome is $R_{1}(t+1)>R_{2}(t+1)$, the value of $h(t+1)$ is positive. Whether it is greater or less than 1 depends on the extent of the change in $\bar{f}(t)$. Larger changes are more likely at low values of $\bar{f}(t)$ (as larger number of bits can be changed from 0 to 1 ).

\footnotetext{
${ }^{10}$ The higher of the two rates of return is greater than 1 , and that the lower of the two is less than 1 .

${ }^{11}$ Note that there is a positive probability that the system reaches the point where $R_{1}(t)=R_{2}(t)$. In this case, a positive rate of mutation will induce changes in the portfolio fractions, increase or decrease its average value, and the inequality of the rates of return will be restored.
} 
If mutation fails to generate sufficient increase in the average portfolio fraction, the direction of inequality is reversed, i.e. $\bar{f}(t+1)<\bar{f}(t)$ and thus $R_{1}(t+1)<R_{2}(t+1)$. In this case, the value of $h(t+1)$ is negative, and whether the absolute value is greater or less than 1 depends on the extent of the change in $R_{1}(t)$ and $R_{2}(t)$, i.e. on the extent of the change in $\bar{f}(t)$. If an increase in $R_{2}(t)$ is greater than a decrease in $R_{1}(t)$, the absolute value of $h(t+1)$ will be greater than 1 and vice versa.

Consider now the case where $R_{1}(t)<R_{2}(t)$ at time $t$. The result of the application of the election operator test at $t+1$ will be $\bar{f}(t+1) \leq \bar{f}(t-1)$. The relationship between $\bar{f}(t+1)$ and $\bar{f}(t)$ will determine the direction of inequality of the rates of return at time $t+1$. There are two possible outcomes at time $t+1$ : that $\bar{f}(t+1)<\bar{f}(t)$ which implies $R_{1}(t+1)<R_{2}(t+1)$, and that $\bar{f}(t+1)>\bar{f}(t)$ which implies $R_{1}(t+1)>R_{2}(t+1)$.

If mutation results in a decrease in the average portfolio fraction sufficient to preserve the direction of inequality, i.e. the outcome is $R_{1}(t+1)<R_{2}(t+1)$, the value of $h(t+1)$ is positive. Again, whether it is greater or less than 1 depends on the magnitude of the change in $\bar{f}(t)$. Larger changes are more likely at high values of $\bar{f}(t)$ because there is a larger number of bits that can be changed from 1 to 0 in order to decrease the value of $\bar{f}(t)$.

If mutation fails to generate sufficient decrease in the average portfolio fraction, the direction of inequality is reversed, i.e $\bar{f}(t+1)>\bar{f}(t)$ and thus $R_{1}(t+1)>R_{2}(t+1)$. In this case, the value of $h(t+1)$ is negative. Whether its absolute value is greater or less than 1 depends again on the extent of the change in $R_{1}(t)$ and $R_{2}(t)$. If an increase in $R_{1}(t)$ is greater than a decrease in $R_{2}(t)$, then $|h(t+1)|>1$. If an increase in $R_{1}(t)$ is less than a decrease in $R_{2}(t)$, then $|h(t+1)|<1$.

Convergence to a stationary equilibrium implies the equality of the rates of return on two currencies as well as the rates of return that are constant over time. The genetic algorithm economy may get to a stationary equilibrium by chance, but it will not stay there and there is a positive probability that an inequality of the rates of return would be restored, resulting in continuing fluctuations of the exchange rate and consequently, fluctuations in the exchange rate returns. Thus the values of the returns on the exchange rate do not tend to settle down to a stationary equilibrium over time. Overall, it is the properties of the map $h(t+1)$, together with the effects of the mutation operator and the boundedness of the set of values of the returns, that give rise to the observed chaotic dynamics.

\section{Conclusions}

We study the overlapping generations economy with two currencies with genetic algorithm learning and investigate the statistical properties of the 
resulting time series data. The theoretical model implies a constant exchange rate under the perfect foresight assumption. Under the genetic algorithm learning, the models equilibrium dynamics is not constant but exhibits bounded oscillations. The time series analysis of the exchange rate returns indicates that the dynamics of the data is chaotic. This result is robust in regard to the parameter values of the economic model and of the genetic algorithm operators. The analysis shows that these dynamics result from the interaction of the properties that characterize the theoretical model and the genetic algorithm adaptation.

\section{Acknowledgements}

We thank Cars Hommes and two anonymous referees for their comments in earlier versions of this paper. Jasmina Arifovic gratefully acknowledges financial support from the Social Sciences and Humanities Research Council of Canada. Ramazan Gençay gratefully acknowledges financial support from the Natural Sciences and Engineering Research Council of Canada and the Social Sciences and Humanities Research Council of Canada.

\section{References}

Arifovic, J., 1995. Genetic algorithms and inflationary economies. Journal of Monetary Economics 36, 219-243.

Arifovic, J., 1996. The behavior of the exchange rate in the genetic algorithm and experimental economies. Journal of Political Economy 104, 510-541.

Arifovic, J., 1998. Stability of equilibria under genetic-algorithm adaptation: an analysis. Macroeconomic Dynamics 2, 1-22.

Arifovic, J., Bullard, J., Duffy, J., 1997. The transition from stagnation to growth: an adaptive learning approach. Journal of Economic Growth 2, 185-209.

Arthur, B., LeBaron, B., Palmer, R., 1999. Time series properties of an artificial stock market. Journal of Economic Dynamics and Control, in press.

Arthur, B., LeBaron, B., Palmer, R., Tayler, P. 1997. Asset pricing under endogenous expectations in an artificial stock market. In: Arthur, B., Durlauf, S., Lane, D. (Eds.), The Economy as an Evolving Complex System II. Addison-Wesley, Reading, MA.

Brock, W.A., Dechert, W.D., Scheinkman, J.A., LeBaron, B., 1996. A test for independence based on the correlation dimension. Econometric Reviews 15, 197-235.

Brock, W.A., Hommes, C.H., 1997. A rational route to randomness. Econometrica 65, 1059-1095.

Brock, W.A., Hommes, C.H., 1999. Heterogeneous beliefs and routes to chaos in a simple asset pricing model. Journal of Economic Dynamics and Control, in press.

Bullard, J., Duffy, J., 1998a. A model of learning and emulation with artificial adaptive agents. Journal of Economic Dynamics and Control 22, 179-207.

Bullard, J., Duffy, J., 1998b. On learning and the stability of cycles. Macroeconomic Dynamics 2, 22-48.

Dawid, H., 1996. Adaptive Learning by Genetic Algorithms. Springer, Berlin. 
Dechert, W.D., Gençay, R., 1992. Lyapunov exponents as a nonparametric diagnostic for stability analysis. Journal of Applied Econometrics 7, 41-60.

Gençay, R., Dechert, W.D., 1992. An algorithm for the $n$ Lyapunov exponents of an $n$-dimensional unknown dynamical system. Physica D 59, 142-157.

Kareken, J., Wallace, N., 1981. On the indeterminacy of equilibrium exchange rates. Quarterly Journal of Economics 96, 207-222.

King, R., Wallace, N., Weber, W., 1992. Nonfundamental uncertainty and exchange rates. Journal of International Economics 32, 83-108.

LeBaron, B., 1997. A fast algorithm for the BDS statistics. Studies in Nonlinear Dynamics and Econometrics 2, 53-59.

Marimon, R., McGrattan, E., Sargent, T.J., 1990. Money as a medium of exchange in an economy with artificially intelligent agents. Journal of Economic Dynamics and Control 14, 329-373. 